\title{
Growth inhibition of human breast carcinoma cells by overexpression of regulator of G-protein signaling 4
}

\author{
HYUN-JUNG PARK, SEUNG-HYUN KIM and DONG-OH MOON \\ Department of Biology Education, Daegu University, Gyeongsan, Gyeongsangbuk-do 38453, Republic of Korea
}

Received April 13, 2015; Accepted July 15, 2016

DOI: $10.3892 / 01.2017 .6009$

\begin{abstract}
Breast cancer remains the second largest cause of mortality in women with cancer and does not respond well to conventional therapies. Regulator of G-protein signaling 4 (RGS4) is a GTPase-activating protein of the heterotrimeric $\mathrm{Gq}$ and $\mathrm{Gi}$ proteins. Altered levels of RGS4 are reportedly linked with several human diseases, including cancer. The present study investigated whether overexpression of RGS4 inhibited the growth of human breast cancer cells. Protein expression was investigated by western blot analysis. Cell viability and apoptosis were analyzed by MTT assay and flow cytometric analysis, respectively. Cell cycle analysis was performed using propidium iodide staining in order to examine the anti-proliferative function of increased RGS4 levels. Next, changes in the expression levels of $\mathrm{G}_{2} / \mathrm{M}$ cell cycle-related proteins were examined. Overexpression of RGS4 led to the upregulation of phosphorylayed (p)-Ser ${ }^{216}$ cell division cycle (Cdc)25C and $\mathrm{p}-\mathrm{Tyr}^{15} \mathrm{Cdc} 2$. Importantly, MG132-induced proteasome blockade prevented degradation of RGS4. Suppression of proliferation was associated with $\mathrm{G}_{2} / \mathrm{M}$-phase cell cycle arrest. Furthermore, enhanced endogenous RGS4 protein levels significantly inhibited breast cancer cell growth, which was reversed by a pharmacological inhibitor of RGS4. Taken together, these results suggest that overexpression of RGS4 in human breast cancer cells by molecular means may offer a potential therapeutic approach.
\end{abstract}

\section{Introduction}

Breast cancer is the most common malignancy among females in the majority of western countries, where women have an overall lifetime risk of $>10 \%$ (1). Breast cancer is a heterogeneous disease sustained by complex growth pathways (1). Previous reports indicate that chemoattractants, including

Correspondence to: Professor Dong-Oh Moon, Department of Biology Education, Daegu University, 201 Daegudae-ro, Gyeongsan-si, Gyeongsan, Gyeongsangbuk-do 38453, Republic of Korea

E-mail: domoon@daegu.ac.kr

Key words: RGS4, $\mathrm{G}_{2} / \mathrm{M}$ cell cycle, breast cancer growth factors, chemokines and matrix metalloproteases, are important in breast cancer cell migration, invasion and growth (2). These ligands bind to membrane receptors on cancer cells, including G-protein coupled receptors (GPCRs), which affect numerous physiological processes, including migration and invasion of breast cancer cells (3). These properties have led to a widespread development of GPCR-targeted drugs, which represent nearly $30 \%$ of all currently used therapeutics, for indications ranging from allergy to depression and hypertension (4). However, the clinical utility of targeting GPCRs in cancer therapy remains poorly defined.

Ligand-activated GPCRs catalyze the exchange of guanosine diphosphate (GDP) for guanosine-5'-triphosphate (GTP) on the $\mathrm{G} \alpha$ subunit, causing the dissociation of the G-protein heterotrimer into G $\alpha$-GTP and G $\beta \gamma$ subunits (5). Subsequently, the two active halves of the G-protein bind and activate various downstream transducer proteins (5). With time, the GTP molecule on G $\alpha$ is hydrolyzed back to GDP; this reaction is strongly stimulated by the group of GTPase-activating protein (GAP) regulators, the majority of which belong to the regulator of G-protein signaling (RGS) family of proteins (6). Proteins of the RGS family modulate GPCR-mediated signals in cells (5). Among the $>20$ RGS proteins known to date, RGS4, a GAP for heterotrimeric $\mathrm{Gq}$ and $\mathrm{Gi}$ (7), is associated with regulation of the hypertrophic response in the failing human myocardium $(8,9)$ and with the regulation of migration and invasion in breast cancer (10). RGS4 has an N-terminal Cys residue that is targeted for ubiquitin-dependent degradation by arginylation through the Arg/N-end rule pathway of protein degradation $(11,12)$. RGS4 negatively regulates $\mathrm{G}$-protein activation by accelerating the hydrolysis of the GTP molecule bound to the $\mathrm{G} \alpha$ subunit and subsequently stabilizing the transition state, and/or by competing for the binding of G-protein to downstream effectors (13). Hypertrophic signaling through $\mathrm{Gq}$ is mediated downstream of the Gaq subunit via several pathways, including calcineurin/nuclear factor of activated $\mathrm{T}$ cells, protein kinase $\mathrm{C}$ and mitogen-activated protein kinases (MAPKs) $(14,15)$. Among the downstream effectors of $\mathrm{G} \beta \gamma$ subunits, phosphoinositide 3-kinase p110 $\gamma$ is of particular interest, due to its role in Akt activation, which ultimately mediates myocyte hypertrophy through the mammalian target of rapamycin complex 1 pathway $(16,17)$. Although dysfunction of RGS4 protein has been reported in various human diseases $(18,19)$, there have been a few studies implicating RGS4 regulation of GPCR signaling in the growth of breast 
cancer cells (20). There is a critical requirement to develop novel strategies for effective prevention and therapy of this disease.

The present study tested the inhibitory effects of RGS4 overexpression on the growth of human breast cancer cells and examined the molecular mechanism by which RGS4 overexpression causes this growth inhibition. The cell cycle analysis data suggested that RGS4 induced $\mathrm{G}_{2} / \mathrm{M}$ arrest, and therefore, $\mathrm{G}_{2} / \mathrm{M}$-related signal transduction pathways associated with breast cancer proliferation were further examined to identify those triggered by RGS4.

\section{Materials and methods}

Cell culture. The human breast cancer cell lines MDA-MB-231 and MCF-7 were purchased from the American Type Culture Collection (Manassas, VA, USA). Cells were cultured in Dulbecco's modified Eagle medium supplemented with 10\% fetal bovine serum (Gibco; Thermo Fisher Scientific, Inc., Waltham, MA, USA) and antibiotics (100 $\mu \mathrm{g} / \mathrm{ml}$ streptomycin and $100 \mathrm{U} / \mathrm{ml}$ penicillin; Thermo Fisher Scientific, Inc.) and maintained at $37^{\circ} \mathrm{C}$ with $5 \% \mathrm{CO}_{2}$ in a humidified atmosphere.

Chemicals. The proteasomal inhibitor MG132 (Sigma-Aldrich; Merck Millipore, Darmstadt, Germany) and the RGS4 inhibitor CCG-63802 [methyl-N-[(4-chlorophenyl) sulfonyl]-4-nitro-benzenesulfinimidoate] (Santa Cruz Biotechnology, Inc., Dallas, TX, USA) were dissolved in anhydrous dimethyl sulfoxide (DMSO; Sigma-Aldrich; Merck Millipore, Darmstadt, Germany), aliquoted and stored at $-80^{\circ} \mathrm{C}$ for the time period indicated by the manufacturer.

Plasmid and transfection. RGS4 complementary (c)DNA was obtained from OriGene Technologies, Inc. (Rockville, MD, USA) and cloned into the mammalian expression vector pcDNA3.1 to generate MC-RGS4. The N-terminal Cys residue targeted for ubiquitin-dependent degradation of RGS4 was mutated to Val (MV-RGS4) using a polymerase chain reaction-based mutagenesis strategy, as previously described (12). Next, the cells were transfected with the aforementioned plasmids using Lipofectamine 2000 reagent (Thermo Fisher Scientific, Inc.) according to the manufacturer's protocol.

Cell viability assays. The viability of cells was examined using the 3-(4,5-dimethylthiazol-2-yl)-2,5-diphenyltetrazolium bromide (MTT) method. Equal numbers of cells in a volume of $200 \mu \mathrm{l}$ were seeded in 48 -well plates $\left(2 \times 10^{4}\right.$ cells/well $)$. Upon transfection, the plates were incubated for 2 days. For each measurement, $50 \mu 1 \mathrm{MTT}(5 \mathrm{mg} / \mathrm{ml})$ was added into each well and incubated at $37^{\circ} \mathrm{C}$ with $5 \% \mathrm{CO}_{2}$ for $2 \mathrm{~h}$. The wells were then decanted, and the purple formazan crystals formed were dissolved in $200 \mu \mathrm{l}$ DMSO. The absorbance of the plate was measured at $595 \mathrm{~nm}$ in an enzyme-linked immunosorbent assay plate reader. All assays were performed in triplicate. An hemocytometer was also used for viable cell counts.

Flow cytometric analysis. Cells $\left(2 \times 10^{5}\right)$ seeded in 6-well plates were transfected with pcDNA3.1 expressing wild type (WT) MC-RGS4 and mutant MV-RGS4. After 2 days of culture, cells wereharvested, and propidium iodide(PI) staining was performed according to a standard method (21). Cell cycle progression was determined using flow cytometry (FACSCalibur; BD Biosciences,Franklin Lakes, NJ,USA), and data analysis was conducted using FlowJo 9.3 software (FlowJo, LLC, Ashland, OR, USA). For annexin- $\mathrm{V}$ staining, live cells were incubated with annexin-V (R\&D Systems, Inc., Minneapolis, MN, USA).

In vitro caspase activity assay. Caspase activity was determined by a colorimetric assay (catalogue no. CASP-3-C; Sigma-Aldrich; Merck Millipore) according to the manufacturer's protocol. Briefly, cells were lysed in the supplied lysis buffer, and the supernatants were collected and then incubated with the supplied reaction buffer containing dithiothreitol and caspase substrate. The reaction was measured by changes in the absorbance at $405 \mathrm{~nm}$ using a microplate reader (Molecular Devices, LLC, Sunnyvale, CA, USA).

Western blotting. Cells were washed with cold phosphate-buffered saline (PBS) and lysed in ice-cold lysis buffer (PRO-PREP ${ }^{\circledR}$; Intron Biotechnology, Inc., Gyeongi-do, Republic of Korea). Protein concentration was determined by the Bradford protein assay (Bio-Rad Laboratories, Inc., Hercules, CA, USA). Protein extracts were resolved on $12 \%$ sodium dodecyl sulfate-polyacrylamide gel electrophoresis and transferred to a polyvinylidene fluoride membrane (EMD Millipore, Billerica, MA, USA). The membrane was blocked in 5\% fat-free milk. The primary antibodies used at a dilution of 1:1,000 were anti- $\alpha$-tubulin (catalogue no. sc-8035; Santa Cruz Biotechnology, Inc.), anti-RGS4 (catalogue no. sc-6204; Santa Cruz Biotechnology, Inc.), rabbit monoclonal anti-human cyclin B1 (catalogue no. 4138; Cell Signaling Technology, Inc., Danvers, MA, USA), rabbit monoclonal anti-human phosphorylated (p)-histone H3 (catalogue no. 53348; Cell Signaling Technology, Inc.), rabbit polyclonal anti-human p-cell division cycle (Cdc)25c (catalogue no. 4901; Cell Signaling Technology, Inc.), rabbit monoclonal anti-human p-Cdc2 (catalogue no. 9116; Cell Signaling Technology, Inc.), rabbit monoclonal anti-human p-B-cell lymphoma-2 (Bcl-2) (catalogue no. 4223; Cell Signaling Technology, Inc.) and rabbit monoclonal antihuman glyceraldehyde 3-phosphate dehydrogenase (GAPDH) (catalogue no. 5174; Sigma-Aldrich; Merck Millipore). The membranes were incubated with the above primary antibodies overnight at $4^{\circ} \mathrm{C}$ to improve the detection limit, and then incubated with anti-rabbit immunoglobulin $\mathrm{G}$ conjugated to horseradish peroxidase secondary antibody at a dilution of 1:2,000 at room temperature for $1 \mathrm{~h}$ upon washing with PBS. Protein signals were visualized with an enhanced chemiluminescence system (Amersham Imager 600; GE Healthcare Life Sciences, Chalfont, UK). GAPDH was used as an equal loading control.

Immunocytochemistry. MDA-MB-231 cells were adhered to poly-D-lysine-coated 12 -well slides and subsequently fixed for $10 \mathrm{~min}$ at room temperature in $4 \%$ paraformaldehyde. Cells were then permeabilized using $0.2 \%$ Triton/Tris-buffered saline (TBS) and blocked with 5\% normal goat serum (catalogue no. I5256; Sigma Aldrich; Merck Millipore) in $0.1 \%$ bovine serum albumin (catalogue no. A2153; Sigma-Aldrich, Merck Millipore)/TBS/Tween 20. Next, cells were incubated overnight at $4^{\circ} \mathrm{C}$ with a mouse antibody against 
A

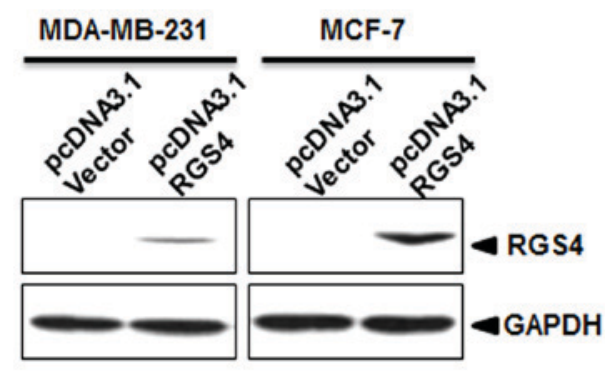

B

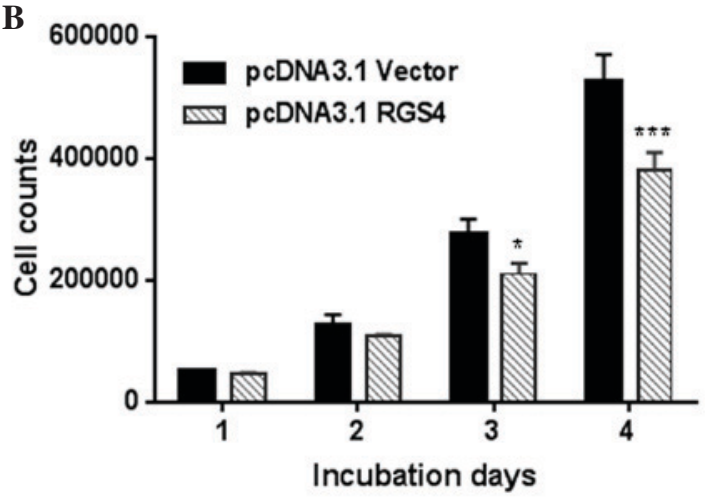

Figure 1. Overexpression of RGS4 reduces cell viability in breast cancer cells. (A) Western blot analysis demonstrated overexpression of wild type RGS4. GAPDH was used as total protein loading control. (B) MDA-MB231 cells (1,000 cells/well) were incubated for 1-3 days, and cell growth was measured with 3-(4,5-dimethylthiazol-2-yl)-2,5-diphenyltetrazolium bromide at the indicated times. The values correspond to relative cell growth and the data are presented as the mean \pm standard deviation. ${ }^{*}<<0.05,{ }^{* * *} \mathrm{P}<0.001$ compared with the vector control. RGS4, regulator of G-protein signaling 4; GAPDH, glyceraldehyde 3-phosphate dehydrogenase.

A

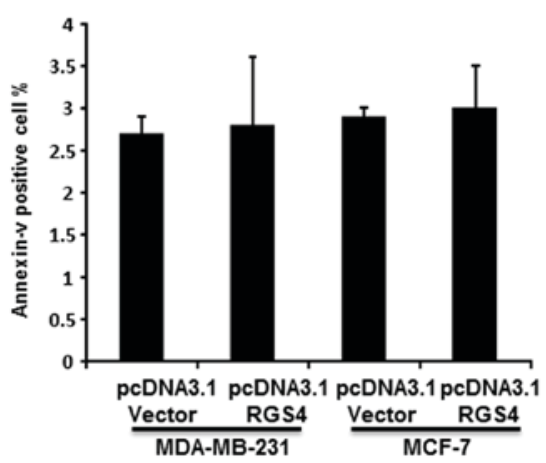

B

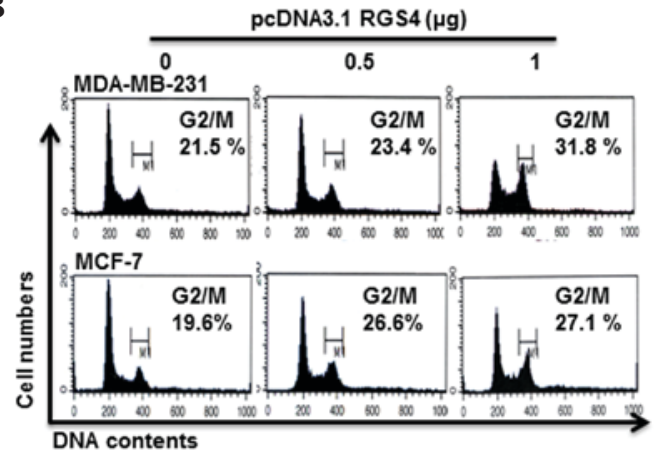

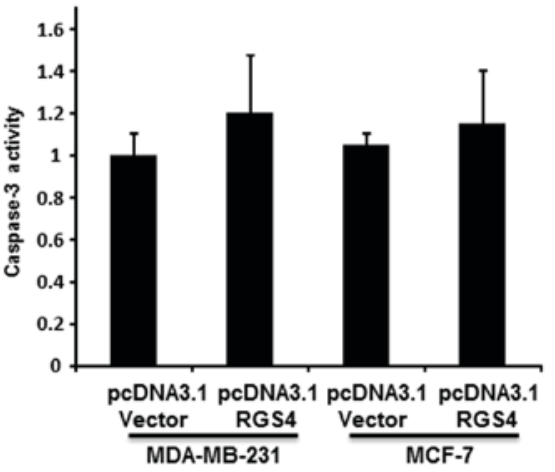

C

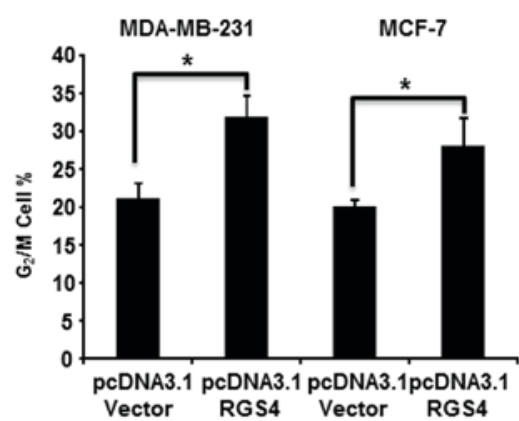

Figure 2. Overexpression of RGS4 leads to $\mathrm{G}_{2} / \mathrm{M}$-phase arrest in breast cancer cells. (A) Flow cytometry analysis of annexin- $\mathrm{V}^{+}$cells and in vitro caspase-3 activity assay. (B) Representative cell cycle histograms with overexpression of RGS4 at 2 days post-transfection. Cells were analyzed by fluorescence-activated cell sorting for DNA content by propidium iodide staining. (C) Bar graphs represent the mean \pm standard deviation of three independent experiments $(* \mathrm{P}<0.05$ compared with the control). RGS4, regulator of G-protein signaling 4 .

$\alpha$-tubulin (catalogue no. T6199; Sigma-Aldrich, Merck Millipore; 1:1,000). Cells were then washed and incubated with an anti-mouse Alexa Fluor ${ }^{\circledR} 488$-conjugated secondary antibody (catalogue no. A11054; Invitrogen; Thermo Fisher Scientific, Inc.; 1:200) to detect antigen-antibody binding. Cells were then stained with Hoechst 33342, washed and mounted using VECTASHIELD ${ }^{\circledR}$ Mounting Medium (Vector Laboratories, Inc., Burlingame, CA, USA).

Statistical analysis. All data are presented as the mean \pm standard deviation of $\geq 3$ independent experiments. Statsitical analysis was performed using GraphPad Prism 6.00 software (GraphPad Software, Inc., La Jolla, CA, USA) Student's t-test was used for the in vitro experiments. Two-way analysis of variance followed by Bonferroni's multiple comparison test was used for analysis of cell viability. $\mathrm{P}<0.05$ was considered to indicate a statistically significant difference.

\section{Results}

Overexpression of RGS4 inhibits cell growth of MDA-MB-231 and $M C F-7$ cells. Protein levels and/or functions of endogenous 
$\mathbf{A}$

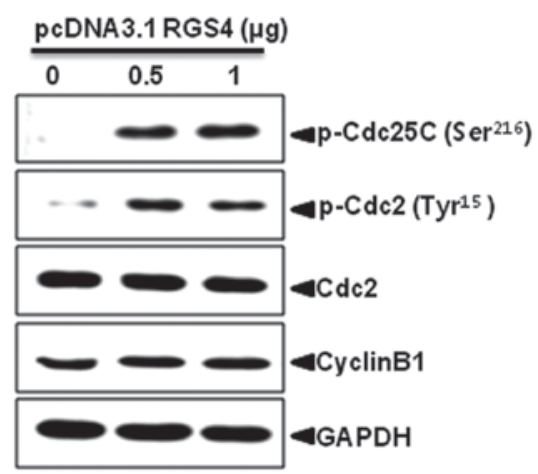

B

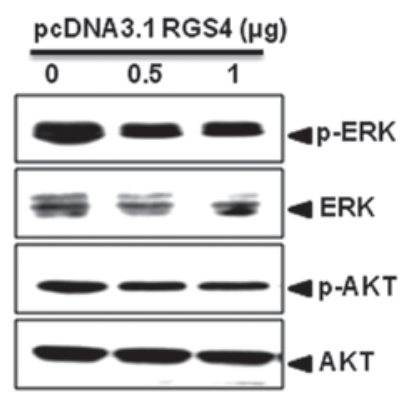

Figure 3. Overexpression of RGS4 induces upregulation of $\mathrm{Ser}^{216} \mathrm{Cdc} 25 \mathrm{C}$ and $\mathrm{p}-\mathrm{Tyr}^{15} \mathrm{Cdc} 2$. (A) Cell lysates were subjected to immunoblotting with the antibodies indicated on the panel. Samples (50 $\mu \mathrm{g}$ protein/lane) were subjected to $10 \%$ sodium dodecyl sulfate-polyacrylamide gel electrophoresis and western blotting for detection of specific proteins, as indicated. GAPDH was used as a loading control. (B) Western blot comparison between RGS4-overexpressing MDA-MB-231 cells and untreated wild type control cells. RGS4, regulator of G-protein signaling 4; GAPDH, glyceraldehyde 3-phosphate dehydrogenase; Cdc, cell division cycle; GAPDH, glyceraldehyde 3-phosphate dehydrogenase; ERK, extracellular signal-regulated kinase; p-, phosphorylated.

A

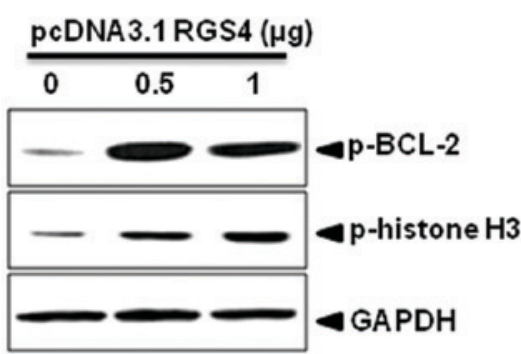

B

a-tubulin-FITC

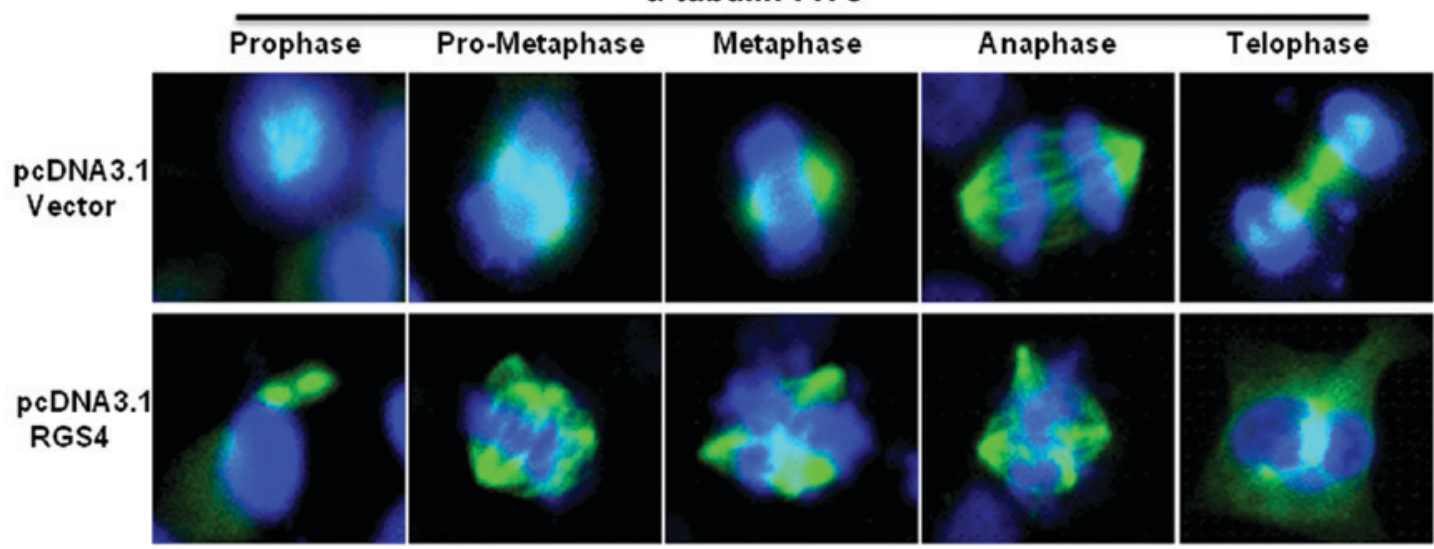

Figure 4. Mitotic arrest induced by RGS4. (A) Immunoblotting of p-histone H3 and Bcl-2 in MDA-MB-231 cells following transfection with RGS4 for $24 \mathrm{~h}$. GAPDH was used as a loading control. (B) Tubulin immunocytochemistry in MDA-MB-231 cells following transfection with RGS4. DNA, blue; tubulin, green. Magnification, x400. RGS4, regulator of G-protein signaling 4; GAPDH, glyceraldehyde 3-phosphate dehydrogenase; p-, phosphorylated; Bcl-2, B-cell lymphoma 2; FITC, fluorescein isothiocyanate.

RGS4 have been previously identified in breast cancer (10). RGS4 becomes unstable by $\mathrm{N}$-arginylation at the oxidized $\mathrm{Cys}^{2}$ residue through a non-ribosomal arginine-transferase, and then is degraded by the proteasome pathway, which is often elevated in breast cancer (10). In the present study, transient expression (50\% transfection efficiency) of RGS4 (WT and mutant) proteins was confirmed by western blotting. The MDA-MB-231 and MCF-7 cell lines, which are human breast cancer cell lines with relatively low levels of endogenous RGS4 expression (10), were selected to evaluate RGS4 overexpression (Fig. 1A). The growth inhibitory effects of RGS4 overexpression on MDA-MB-231 and MCF-7 cell lines was then examined using MTT assay. As indicated in Fig. 1B, overexpression of RGS4 inhibited the growth of MDA-MB-231 cells. Cell growth of MCF-7 cells overexpressing RGS4 was reduced by $26.6 \%$. These results indicated that RGS4 significantly inhibited cell growth in both cell lines (MDA-MB231, $\mathrm{P}=0.011$ and 0.002 , respectively; MCF-7, $\mathrm{P}=0.031$ and 0.002 , respectively, for pcDNA3.1 RGS4 doses 0.5 and $1 \mu \mathrm{g}$ ), which was consistent with previously published results reporting 


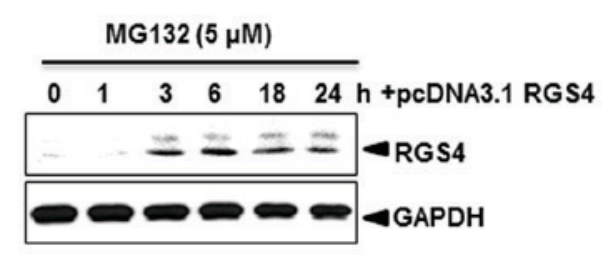

B

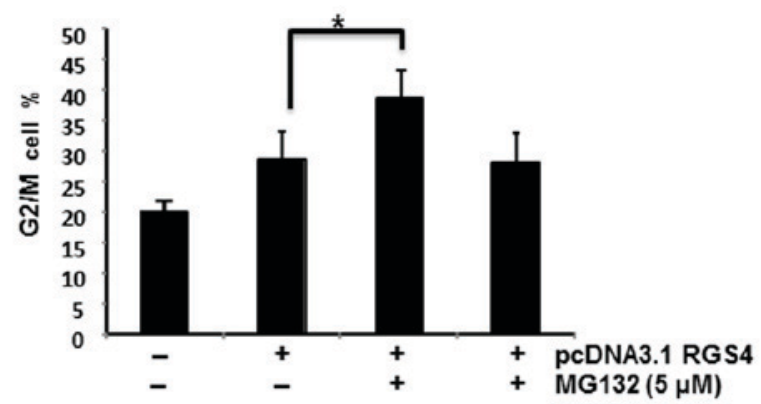

C

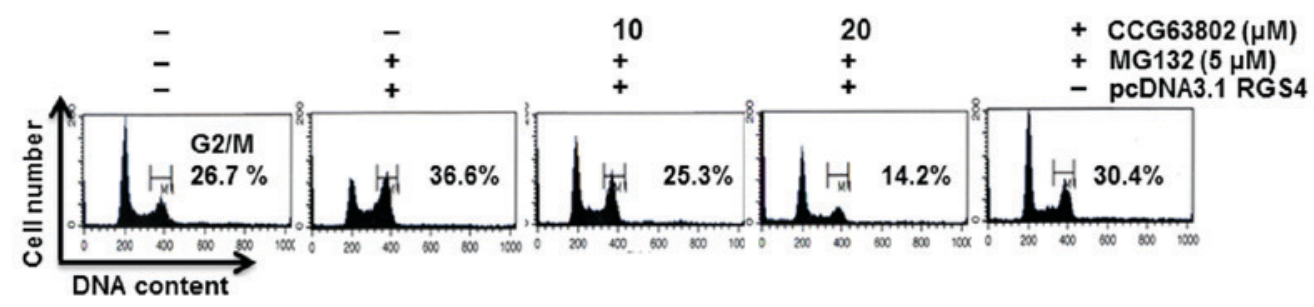

Figure 5. MG132 enhanced RGS4-mediated cell cycle arrest. (A) MDA-MB-231 cells were stimulated with $5 \mu$ M MG132 for 24 h following RGS4 transfection, and then cell lysates were extracted. Equal amounts of cell lysates (50 $\mu \mathrm{g})$ were resolved by sodium dodecyl sulfate-polyacrylamide gel electrophoresis, transferred to polyvinylidene fluoride membranes and probed with an specific antibody against RGS4. GAPDH was used as a loading control. (B) MDA-MB-231 cells were pretreated with $5 \mu \mathrm{M}$ MG132 for $24 \mathrm{~h}$ following RGS4 transfection. Cells were analyzed by fluorescence-activated cell sorting for DNA content by propidium iodide staining. The percentage of $\mathrm{G}_{2} / \mathrm{M}$-phase cells was determined based on a DNA content histogram. The results are expressed as the percentage of the vehicle control value. Data are the mean \pm standard deviation values from three independent experiments (*P<0.05). (C) MDA-MB-231 cells were treated with $5 \mu \mathrm{M}$ MG132 and transfected with RGS4 for $24 \mathrm{~h}$. Then, their cell cycle distribution was analyzed by flow cytometry. RGS4, regulator of G-protein signaling 4; GAPDH, glyceraldehyde 3-phosphate dehydrogenase.

that overexpressed RGS4 inhibits the growth of breast cancer cells (10).

RGS4 overexpression induces $G_{2} / M$-phase cell cycle arrest. Annexin-V and caspase-3 activity assays were conducted to determine whether the inhibitory effect of RGS4 overexpression was associated with induction of apoptosis or reduction of cell proliferation in breast cancer cells. No difference in apoptosis was detected between the untreated WT control group and MDA-MB-231 cells with RGS4 overexpression (Fig. 2A and B). Cell cycle progression analysis was performed by fluorescence-activated cell sorting scanning using PI staining to investigate the mechanism by which RGS4 overexpression induces reduction in breast cancer cell proliferation. In both MDA-MB-231 and MCF-7 cells, RGS4 overexpression caused an increase in the proportion of cells in $G_{2} / M$ phase and a decrease in the cell population in $G_{1}$ phase, as compared with the untreated WT control groups, which indicated a $\mathrm{G}_{2} / \mathrm{M}$-phase cell cycle arrest (Fig. 2C). Specifically, the percentage of cell population in $G_{2} / M$ phase of MDA-MB-231 cells increased from 21.5 to $31.8 \%$, and in MCF-7 cells, it increased from 19.6 to $27.1 \%$. These results indicated that overexpression of WT RGS4 led to arrest in the $\mathrm{G}_{2} / \mathrm{M}$ phase of the cell cycle in breast cancer cells.

RGS4 overexpression upregulates $p$-Ser ${ }^{216}$ Cdc25C and $p$-Tyr ${ }^{15}$ $C d c 2$. Several cell cycle-relevant proteins were screened for changes in their expression level and phosphorylation status to further determine the molecular events that are involved in the observed $\mathrm{G}_{2} / \mathrm{M}$-phase cell cycle arrest. As represented in Fig. 3A, dose-dependent increased levels of p-Ser ${ }^{216} \mathrm{Cdc} 25 \mathrm{C}$ and $\mathrm{p}-\mathrm{Tyr}^{15} \mathrm{Cdc} 2$ (inactive) were detected in response to exogenous RGS4 expression. Cyclin B1 is also important for Cdc2 activity (22); however, its levels remained unchanged following transfection with RGS4. Additionally, to determine how RGS4 regulates signal transduction pathways to inhibit cell cycle transition, activation of the extracellular signal-regulated kinase (ERK) and Akt signaling pathways was studied, since both are known regulators of breast cancer proliferation (23). As indicated in Fig. 3B, RGS4 overexpression inactivated ERK but did not affect Akt phosphorylation.

RGS4 induces mitotic arrest. Next, it was determined whether RGS4 overexpression caused arrest of cells in the $G_{2}$ or $M$ phases. As represented in Fig. 4A, immunoblotting demonstrated that the mitotic triggers, histone $\mathrm{H} 3$ and $\mathrm{Bcl}-2$, were hyperphosphorylated. These findings clearly demonstrated that RGS4 overexpression arrested the cells in M phase. The fraction of MDA-MB-231 cells with mitotic spindles was determined by visualization of tubulin immunofluorescence and Hoechst staining, in order to characterize and verify the effect of RGS4 on mitosis. Tubulin immunocytochemistry revealed that microtubules appeared star-shaped and surrounded by clusters of DNA, which was suggestive of a prometaphase arrest of RGS4 overexpressing-cells (Fig. 4B).

MG132 enhances RGS4-induced $G_{2} / M$ arrest. RGS4 is subjected to proteasomal degradation, and treatment with proteasome inhibitors increases RGS4 protein levels $(24,25)$. Accordingly, it was determined whether treatment with the proteasomal inhibitor MG132 could increase RGS4 expression via blockage of proteasomal degradation in 
RGS4-overexpressing MDA-MB-231 cells. As indicated in Fig. 5A, overnight treatment of RGS4-overexpressing MDA-MB-231 cells with the proteasome inhibitor MG132 $(5 \mu \mathrm{M})$ dramatically increased the RGS4 protein levels by $>3$-fold. These results demonstrated that the RGS4 protein levels were regulated by proteasome degradation. MG132 also effectively enhanced the $\mathrm{G}_{2} / \mathrm{M}$-phase cell population in RGS4-overexpressing cells (Fig. 5B). Notably, transfection with N-terminal mutated (Cys $\rightarrow$ Val) RGS4 (MV-RGS4) was more stable and induced $\mathrm{G}_{2} / \mathrm{M}$ cell cycle arrest more effectively than transfection with non-mutated RGS4 (MC-RGS4) did (data not shown). It was also observed that CCG-63802, a compound inhibitor of RGS4, significantly blocked RGS4-mediated $\mathrm{G}_{2} / \mathrm{M}$ cell cycle arrest $(\mathrm{P}=0.0003 ;$ Fig. 3C).

\section{Discussion}

RGS proteins provide a critical point of control for numerous cellular processes and signaling cascades initiated by inhibitory G-proteins (6,7). During GPCR activation, GDP is exchanged for GTP on the Ga subunit, which then releases the $G \alpha$ and $\beta \gamma$ subunits to modulate their effectors, including adenylyl cyclase, ion channels and phospholipase C (5). RGS proteins are GAPs that markedly increase the rate of the $\mathrm{G} \alpha$ subunit's GTP hydrolysis and function to reduce the magnitude and duration of $\mathrm{G} \alpha$ and $\mathrm{G} \beta \gamma$ signals $(6,7)$. Previous studies demonstrated a complete lack of correlation between RGS4 messenger RNA and protein levels in metastatic breast cancer cells due to its rapid proteasomal degradation (10). Although it has been reported that RGS4 facilitates breast cancer invasiveness and growth both in vitro and in vivo, its function on the cell cycle has not been explored thus far (8). In the present study, breast cancer cell lines were transfected with a recombinant cDNA plasmid containing the open reading frame of RGS4. The results demonstrated that exogenous RGS4 significantly inhibited the growth of breast cancer cells. However, ectopic RGS4 could not induce cell apoptosis in MDA-MA-231 or MCF-7 cells, but it arrested cells in $\mathrm{G}_{2} / \mathrm{M}$ phase.

Cell cycle arrest in RGS4-transfected cells was accompanied by a marked increase in phosphorylation of $\mathrm{Cdc} 2$ and $\mathrm{Cdc} 25 \mathrm{C}$. It is reasonable to postulate that RGS4 may regulate the activity of $\mathrm{Cdc} 2 /$ cyclin $\mathrm{B} 1$ kinase through reducing this complex formation by causing the accumulation of $\mathrm{p}-\mathrm{Thr}^{14} / \mathrm{Tyr}^{15} \mathrm{Cdc} 2$ (inactive). Western blotting using an anti-p-Cdc2 antibody revealed an increase in the level of p-Tyr ${ }^{15} \mathrm{Cdk} 1$ in RGS4-transfected cells. The activity of $\mathrm{Cdc} 25 \mathrm{C}$ is negatively regulated by phosphorylation at $\mathrm{Ser}^{216}$, which creates a binding site for 14-3-3 (26,27). The binding with 14-3-3 hinders nuclear accumulation of Cdc25C, which is required for the activation of the nuclear $\mathrm{Cdc} 2 /$ cyclin $\mathrm{B} 1$ kinase complex (28). Therefore, phosphorylation of Cdc25C on $\mathrm{Ser}^{216}$ represents an important regulatory mechanism by which cells delay or block mitotic entry under normal conditions and in response to DNA damage $(26,27)$. RGS4-transfected cells exhibited an increase in Ser ${ }^{216}$ phosphorylation of $\mathrm{Cdc} 25 \mathrm{C}$, which persisted for the duration of the experiment ( $48 \mathrm{~h}$ after RGS4 transfection). Additionally, it has been reported that the MAPK pathway is involved in $\mathrm{G}_{2} / \mathrm{M}$ progression (29). It is known that ERK inhibition causes myelin transcription factor 1 activation, which inhibits Cdc2 (30). Furthermore, it has been reported that the cyclin $\mathrm{B} / \mathrm{Cdc} 2$ complex is retained in the cytosol when ERK is inhibited, and this inhibition of Cdc2 leads to $\mathrm{G}_{2} / \mathrm{M}$-phase arrest (31). The present study demonstrated that RGS4 transfection induced a decrease in ERK1/2 phosphorylation. However, the precise mechanism of the cascade, which is triggered by RGS4-mediated ERK inhibition and leads to $\mathrm{G}_{2} / \mathrm{M}$ arrest, has not been fully elucidated in our system. Thus, further studies are required to clarify the mechanism underlying $\mathrm{G}_{2} / \mathrm{M}$ arrest following ERK inhibition. In addition, immunoblotting revealed that RGS4 overexpression caused mitotic arrest, as demonstrated by hyperphosphorylation of Bcl-2 and histone H3. These findings were clear evidence that RGS4 overexpression arrested cells in M phase.

RGS4 is an unstable protein that is subjected to the $\mathrm{N}$-end rule pathway; thus, removal of the $\mathrm{N}$-terminal Met and enzymatic arginylation of the resulting $\mathrm{N}$-terminal Cys by arginine transferase promotes its ubiquitination and proteasomal degradation $(11,12)$. In the present study, the proteasome inhibitor MG132 increased the basal level of RGS4 and enhanced RGS4-mediated $\mathrm{G}_{2} / \mathrm{M}$ cell cycle arrest, which may have important implications in RGS4 function. Notably, proteasome activity is often elevated in breast cancer (32). Preventing RGS4 degradation by inhibiting the proteasome activity should retard breast cancer cell growth, thus facilitating cancer therapy. The present study demonstrated the growth inhibitory effects of RGS4 overexpression in breast cancer cells. This anti-proliferative role of elevated RGS4 functions in a cell cycle-dependent manner. The molecular mechanisms by which altered levels of RGS4 regulate and orchestrate cellular processes deserve to be explored further for a better understanding of the role of RGS4 in cancer cells. Overall, the present results are suggestive of a possible therapeutic approach for the treatment of human breast cancer by increasing the level of RGS4 in human breast cancer cells.

\section{Acknowledgements}

The present study was partly supported by a Daegu University Research Grant (Gyeongsan, Korea) awarded in 2013.

\section{References}

1. Feuer EJ, Wun LM, Boring CC, Flanders WD, Timmel MJ and Tong T: The life time risk of developing breast cancer. J Natl Cancer Inst 85: 892-897, 1993.

2. Condeelis J, Singer RH and Segall JE: The great escape: When cancer cells hijack the genes for chemotaxis and motility. Annu Rev Cell Dev Biol 21: 695-718, 2005.

3. Dorsam RT and Gutkind JS: G-protein-coupled receptors and cancer. Nat Rev Cancer 7: 79-94, 2007.

4. Feigin ME: Harnessing the genome for characterization of G-protein coupled receptors in cancer pathogenesis. FEBS J 280: 4729-4738, 2013 .

5. Gilman AG: G proteins: Transducers of receptor-generated signals. Annu Rev Biochem 56: 615-649, 1987.

6. Ross EM and Wilkie TM: GTPase-activating proteins for heterotrimeric $\mathrm{G}$ proteins: Regulators of $\mathrm{G}$ protein signaling (RGS) and RGS-like proteins. Annu Rev Biochem 69: 795-827, 2000.

7. Hao J, Michalek C, Zhang W, Zhu M, Xu X and Mende U: Regulation of cardiomyocyte signaling by RGS proteins: Differential selectivity towards $\mathrm{G}$ proteins and susceptibility to regulation. J Mol Cell Cardiol 41: 51-61, 2006. 
8. Mittmann C, Chung CH, Höppner G, Michalek C, Nose M, Schüler C, Schuh A, Eschenhagen T, Weil J, Pieske B, et al: Expression of ten RGS proteins in human myocardium: Functional characterization of an upregulation of RGS4 in heart failure. Cardiovasc Res 55: 778-786, 2002.

9. Owen VJ, Burton PB, Mullen AJ, Birks EJ, Barton P and Yacoub MH: Expression of RGS3, RGS4 and Gi alpha 2 in acutely failing donor hearts and end-stage heart failure. Eur Heart J 22: 1015-1020, 2001.

10. Xie Y, Wolff DW, Wei T, Wang B, Deng C, Kirui JK, Jiang H, Qin J, Abel PW and Tu Y: Breast cancer migration and invasion depend on proteasome degradation of regulator of G-protein signaling 4. Cancer Res 69: 5743-5751, 2009.

11. Lee PC, Sowa ME, Gygi SP and Harper JW: Alternative ubiquitin activation/conjugation cascades interact with $\mathrm{N}$-end rule ubiquitin ligases to control degradation of RGS proteins. Mol Cell 43: 392-405, 2011.

12. Davydov IV and Varshavsky A: RGS4 is arginylated and degraded by the N-end rule pathway in vitro. J Biol Chem 275: 22931-22941, 2000.

13. Popov S, Yu K, Kozasa T and Wilkie TM: The regulators of $\mathrm{G}$ protein signaling (RGS) domains of RGS4, RGS10, and GAIP retain GTPase activating protein activity in vitro. Proc Natl Acad Sci USA 94: 7216-7220, 1997.

14. Hubbard KB and Hepler JR: Cell signalling diversity of the Gqalpha family of heterotrimeric G proteins. Cell Signal 18: 135-150, 2006

15. Tirziu D and Simons M: Endothelium-driven myocardial growth or nitric oxide at the crossroads. Trends Cardiovasc Med 18: 299-305, 2008.

16. Van Berlo JH, Maillet M and Molkentin JD: Signaling effectors underlying pathologic growth and remodeling of the heart. J Clin Invest 123: 37-45, 2013

17. Dupré DJ, Robitaille M, Rebois RV and Hébert TE: The role of Gbetagamma subunits in the organization, assembly, and function of GPCR signaling complexes. Annu Rev Pharmacol Toxicol 49: 31-56, 2009.

18. Levitt P, Ebert P, Mirnics K, Nimgaonkar VL and Lewis DA: Making the case for a candidate vulnerability gene in schizophrenia: Convergent evidence for regulator of G-protein signaling 4 (RGS4). Biol Psychiatry 60: 534-537, 2006.

19. Tekumalla PK, Calon F, Rahman Z, Birdi S, Rajput AH, Hornykiewicz O, Di Paolo T, Bédard PJ and Nestler EJ: Elevated levels of DeltaFosB and RGS9 in striatum in Parkinson's disease. Biol Psychiatry 50: 813-816, 2001.
20. Kelly P, Moeller BJ, Juneja J, Booden MA, Der CJ, Daaka Y, Dewhirst MW, Fields TA and Casey PJ: The G12 family of heterotrimeric $\mathrm{G}$ proteins promotes breast cancer invasion and metastasis. Proc Natl Acad Sci USA 103: 8173-8178, 2006.

21. Riccardi $C$ and Nicoletti I: Analysis of apoptosis by propidium iodide staining and flow cytometry. Nat Protoc 1: 1458-1461, 2006.

22. Wang TH, Wang HS and Soong YK: Paclitaxel-induced cell death: Where the cell cycle and apoptosis come together. Cancer 88: 2619-2628, 2000.

23. O'Reilly KE, Rojo F, She QB, Solit D, Mills GB, Smith D, Lane H, Hofmann F, Hicklin DJ, Ludwig DL, et al: mTOR inhibition induces upstream receptor tyrosine kinase signaling and activates Akt. Cancer Res 66: 1500-1508, 2006.

24. Bodenstein J, Sunahara RK and Neubig RR: N-terminal residues control proteasomal degradation of RGS2, RGS4 and RGS5 in human embryonic kidney 293 cells. Mol Pharmacol 71: 1040-1050, 2007.

25. Krumins AM, Barker SA, Huang C, Sunahara RK, Yu K, Wilkie TM, Gold SJ and Mumby SM: Differentially regulated expression of endogenous RGS4 and RGS7. J Biol Chem 279: 2593-2599, 2004.

26. Sanchez Y, Wong C, Thoma RS, Richman R, Wu Z, Piwnica-Worms $\mathrm{H}$ and Elledge SJ: Conservation of the Chk1 checkpoint pathway in mammals: Linkage of DNA damage to Cdk regulation through Cdc25. Science 277: 1497-1501, 1997.

27. Peng CY, Graves PR, Thoma RS, Wu Z, Shaw AS and Piwnica-Worms H: Mitotic and G2 checkpoint control: Regulation of 14-3-3 protein binding by phosphorylation of $\mathrm{Cdc} 25 \mathrm{C}$ on serine-216. Science 277: 1501-1505, 1997.

28. Kumagai A and Dunphy WG: Binding of 14-3-3 proteins and nuclear export control the intracellular localization of the mitotic inducer Cdc25. Genes Dev 13: 1067-1072, 1999.

29. Dumesic PA, Scholl FA, Barragan DI and Khavari PA: Erk1/2 MAP kinases are required for epidermal G2/M progression. J Cell Biol 185: 409-422, 2009.

30. Palmer A, Gavin AC and Nebreda AR: A link between MAP kinase and p34(cdc2)/cyclin B during oocyte maturation: p90(rsk) phosphorylates and inactivates the p34(cdc2) inhibitory kinase Myt1. EMBO J 17: 5037-5047, 1998.

31. Chambard JC, Lefloch R, Pouysségur J and Lenormand P: ERK implication in cell cycle regulation. Biochim Biophys Acta 1773: 1299-1310, 2007.

32. Chen L and Madura K: Increased proteasome activity, ubiquitin-conjugating enzymes, and eEF1A translation factor detected in breast cancer tissue. Cancer Res 65: 5599-5606, 2005. 